\section{Angiosperm polyploids and their road to evolutionary success}

\author{
Jeffrey A. Fawcett, ${ }^{1,2}$ Yves Van de Peer ${ }^{1,2}$ \\ 'Department of Plant Systems Biology, \\ Flanders Institute for Biotechnology \\ (VIB), Belgium; \\ ${ }^{2}$ Department of Molecular Genetics, Gent \\ University, Belgium
}

\section{Abstract}

The abundance of polyploidy among flowering plants has long been recognized, and recent studies have uncovered multiple ancient polyploidization events in the evolutionary history of several angiosperm lineages. Once polyploids are formed they must get locally established and then propagate and survive while adapting to different environments and avoiding extinction. This might ultimately lead to their long-term evolutionary success, where their descendant lineages survive for tens of millions of years. Along this road to evolutionary success, polyploids must overcome several obstacles, to which several genetic and ecological factors are likely to contribute. One recurrent observation, based on present-day polyploids, has been the high frequency of polyploids in harsh environments. Also, recent studies proposed that the success of certain ancient polyploids might be linked to periods of climatic change. Although we are still in the early stages of unraveling the factors that resulted in the long-term evolutionary success of ancient polyploids, the advances in genomic sequencing and molecular dating methods promise to enhance our understanding. It, therefore, seems timely to review our current knowledge of what determines the success of polyploids. Here, we discuss especially how harsh conditions or periods of climatic change might affect the rate of formation, establishment, persistence and long-term evolutionary success of polyploids in angiosperms.

\section{The polyploid nature of angiosperms}

The genomic data accumulating from various species shows that most angiosperms have undergone one or more polyploidizations, or whole-genome duplications (WGDs), in their evolutionary history. ${ }^{1.5}$ This means that on several different occasions, a newly established polyploid species or population managed to persist long enough to become an evolutionarily successful lineage, in the sense that at least one or more extant species descended from that polyploid ancestor so that we can observe traces of that polyploidy event within the genomes of extant species. The question is what determined the evolutionary success of these polyploids, and why did these particular polyploids attain evolutionary success.

The process that leads to the evolutionary success of polyploids can be characterized by the following phases (Figure 1): the formation of individual polyploids, followed by their establishment and subsequent persistence, ultimately leading to their long-term evolutionary success. ${ }^{6,7}$ Although this is a continuous process and there are no clear boundaries between each phase, we will loosely define them for the sake of clarity throughout the article. Once individual polyploids are formed, they have to get locally established, meaning that a number of polyploids have undergone successive generations and exist as a viable, independent population, reproductively isolated from their parental taxa. The persistence of polyploids can be viewed as a phase which involves their further propagation and survival by, in some cases, adapting to different environments. However, not all polyploids that persist will attain long-term evolutionary success because of a background extinction rate causing species or taxa to disappear due to various factors such as competition or climatic changes. Long-term evolutionary success will be defined here as survival of a polyploidderived lineage for tens of millions of years, regardless of the number of species it gave rise to. Polyploidy can be generally considered to be disadvantageous ${ }^{8}$ and polyploids often exhibit lower fertility and reduced fitness compared to their diploid progenitors. ${ }^{9}$ In addition, their mating opportunities are often limited because triploids that are formed by crosses between tetraploids and diploids are thought to be less viable and fertile compared to diploids, ${ }^{9}$ and the less frequent cytotypes within a population are more likely to get excluded. ${ }^{10}$ Despite these various disadvantages and obstacles that polyploids have to overcome, their local establishment and short-term persistence is not such a rare event, and polyploids have managed to attain long-term evolutionary success on some occasions during the evolutionary past of angiosperms. Several articles have reviewed the different ecological and genetic factors regarding the formation and the subsequent establishment and persistence of polyploids. ${ }^{6,711-14}$ One frequently cited observation is that polyploids are often abundant in harsh and unstable environments, and that they often occupy new niches in which their diploid progenitors could not survive. For instance, polyploids have been reported to be abundant at high latitudes and high altitudes, ${ }^{11,12}$ a wellknown example being the high frequency of polyploids in arctic areas. ${ }^{15,16}$ So what are the
Correspondence: Yves Van de Peer, Gent University, 9052 Gent, Belgium E-mail: yves.vandepeer@psb.vib-ugent.be

Key words: polyploidy, angiosperms, CretaceousTertiary boundary, extinction, genome duplication.

Acknowledgments: the research of JAF and YVdP was supported in part by the European Commission through the 6th Framework Programme (contract number: FOOD-CT-2006 016214) and IUAP P6/25 (Bio-MaGNet). We thank anonymous reviewers for their helpful comments.

Received for publication: 27 December 2009.

Revision received: 20 April 2010.

Accepted for publication: 21 April 2010.

This work is licensed under a Creative Commons Attribution 3.0 License (by-nc 3.0).

(C) Copyright J.A. Fawcett and Y. Van de Peer, 2010 Licensee PAGEPress, Italy

Trends in Evolutionary Biology 2010; 2:e3 doi:10.4081/eb.2010.e3

different factors that might explain the apparent association between polyploidy and environmental changes or harsh conditions, and how might these affect the chances for polyploids to get established and persist?

\section{Establishment and persistence of polyploids}

\section{Non-adaptive, mechanistic factors}

Various mechanistic factors can influence the chances of polyploids getting established and persisting in unstable and harsh environments. The first step in the establishment of polyploids is the formation of individual polyploids. Polyploids often form when unreduced (2n) gametes are produced due to errors during meiosis, ${ }^{9}$ and the number of unreduced gametes can increase under fluctuating conditions, such as large changes in temperature, or on exposure to cold. ${ }^{17}$ This will lead to a higher number of polyploids within a population, which will increase their chance of mating. It has been suggested that the high frequency of polyploids under harsh conditions might be because of the increase in the production of unreduced gametes under stress. ${ }^{9}$ In addition, the population size is likely to have a large effect on the establishment of polyploids. ${ }^{6}$ The chance for a random trait to sweep and take over the population will become much higher if the population size is small, and it has been suggested that the chance effects that occur in small populations could be the most determinant factors in the establishment of polyploids. ${ }^{6}$ 
Under extreme conditions, in which many individuals cannot survive, the population is expected to be small, and the chances of polyploids propagating and becoming established might become much bigger.

Polyploidy has often been linked to different reproductive strategies such as selfing, asexual reproduction, and perennial life-cycle, which might also affect their establishment and persistence. ${ }^{18}$ A recent study that used data from 235 species of flowering plants found that polyploids often have a higher rate of self-fertilization than their diploid relatives. ${ }^{19}$ Their ability to self or to propagate clonally would allow the polyploids to reproduce and propagate without mating partners and a perennial life-cycle will provide them with a longer opportunity to find mating partners of the same cytotype. ${ }^{11}$ These reproductive strategies might provide a competitive advantage when mating opportunities are scarce in unfriendly environments in which it is difficult for most individuals to survive, or upon dispersal to new niches that become available under periods of environmental change.

Periods of rapid climatic change would result in various changes in the ecosystem, which would free and make available many niches because several species which had adapted to local, specific environments are likely to become extinct..$^{20}$ The lack of available niches can limit the successful establishment of polyploids. It was observed that the recently formed allopolyploid plant York groundsel (Senecio eboracensis) is facing extinction because it has not been able to colonize new niches. ${ }^{21}$ Thus, an increase in the number of empty, available niches would increase the possibility for newly formed polyploids to expand their distribution and colonize new niches without facing competition from their diploid parents. Also, Dynesius and Jansson ${ }^{22}$ predicted that periods of climatic change would select for generalists with high vagility (dispersal ability and propensity) rather than specialists that adapt to very specific, local environments. They suggested that this would work against gradual speciation (caused by gradual changes over many generations), and result in a higher proportion of species formed by abrupt mechanisms such as polyploidy. ${ }^{22}$

\section{Adaptive, genetic factors}

The doubling, or merging, of genomes can result in genomic rearrangements, ${ }^{23}$ epigenetic reprogramming, ${ }^{24}$ and changes in expression pattern, ${ }^{25}$ resulting in polyploids that can differ largely from their diploid parents both in genotype and in phenotype. Although many of these changes are likely to be disadvantageous, ${ }^{8}$ especially under stable conditions and in the original environment to which the diploid progenitors are presumably well adapted, a changed environment might result in polyploid individuals having a selective advan- tage. ${ }^{13}$ The fact that polyploids have an extra set of genes has been proposed to result in various adaptive advantages. Having an extra copy can allow genes to take on new functions without altering the original gene function, whereas duplicated genes can also have a wider range of expression patterns. ${ }^{26}$ Polyploids are more likely to have increased heterozygosity and can achieve a much higher number of combinations of alleles, while novel combinations of gene regulatory factors might also arise. ${ }^{13,27} \mathrm{~A}$ recent study showed that this could lead to polyploids being more resistant to pathogens compared to their diploid progenitors. ${ }^{28}$ Such potential advantages are especially pronounced when polyploids are allopolyploids and are hybrids between two distantly related individuals, or if they are formed recurrently. ${ }^{29}$

Many of the factors discussed above, and possibly many others, can potentially influence the successful establishment and persistence of polyploids. These need not be mutually exclusive, and it is likely that the combination of these factors will enhance the chances for polyploids to get established. The main question remains how these factors interact and contribute to the establishment of polyploids under different conditions. Some studies have attempted to address this question by modeling the establishment and persistence process of newly formed polyploids. More than a decade ago, Felber ${ }^{30}$ studied the rate of unreduced gamete production necessary for tetraploids to outcompete diploids under different fertility and viability. The author concluded that the conditions for the establishment of polyploids are rather restrictive, and that a large increase in the unreduced gamete production induced by environmental or genetic changes, or chance processes in small populations, would be required in most cases. Some recent studies have extended these results by accounting for various additional parameters. Li et al.$^{31}$ suggested that the mode of seed/pollen dispersal and the heterogeneity of the environment have a considerable effect on the rate of unreduced gamete production required for polyploids to become established. Building upon the results of Li et al.., ${ }^{31}$ Baack ${ }^{32}$ suggested that higher rates of self-fertilization and higher competitive advantage both lead to an increase in the probability of tetraploid persistence, and also that shorter dispersal distance of seed and pollen increases the probability. Rausch and Morgan $^{33}$ showed the importance of small population size and low inbreeding depression in tetraploids. These studies imply that several factors can lead to the establishment and persistence of polyploids, and further efforts in modeling, while accounting for various conditions, is likely to further enhance our understanding of the establishment and persistence of polyploids.
Do polyploids have a competitive advantage over their diploid progenitors?

The genetic characteristics of polyploids discussed above can, in theory, provide polyploids with an intrinsic advantage over diploids and enable them to more successfully colonize new environments. However, does this mean that polyploids have an intrinsic competitive advantage over diploids, or are they better able to adapt to and colonize harsh environments compared to their diploid progenitors? So far, it has been difficult to prove that polyploids in general have a higher adaptability or ecological tolerance compared to their diploid progenitors. For instance, Martin and Husband ${ }^{34}$ compared the geographical and ecological ranges of polyploids and diploids taking their phylogenetic history into account, but could not find any significant differences in the ranges of polyploids and their diploid relatives. Another recent study reported an example in which diploids had a higher fitness and are replacing polyploids. ${ }^{35}$ The supposed link between polyploids and harsh environments can often, at least partly, be explained by mechanistic factors discussed above, such as the rate of unreduced gamete production or reproductive mechanisms. ${ }^{9,18}$ The abundance of polyploids in arctic regions is probably partly due to the abundance of selfers, ${ }^{18}$ and the abundance of polyploids in higher latitudes could be largely due to the large proportion of perennials in such regions. ${ }^{36}$ Compared to normal conditions in which the diploids are expected to have a much higher fitness and higher frequency than polyploids, the change in environment and/or migration to new niches would increase the relative fitness of polyploids over diploids, and increase the frequency of polyploid individuals, which would lead to a higher probability for polyploids to become established and persist than under normal conditions. Also, if the only niches available are harsh environments, the fraction of polyploids would consequently be higher there, although the polyploids that did successfully colonize such environments were probably those that succeeded in adapting, and their duplicated nature might have been utilized for their successful adaptation. Thus, although theoretical studies and various observations suggest that polyploids do have a better chance to get established under harsh conditions or climatic changes than under normal circumstances, it is important to note that this is not equal to saying that polyploids are more adaptive than diploids.

\section{Long-term evolutionary success of polyploids}

So far, we have discussed the different fac- 
tors that can determine the establishment and persistence of polyploids. We have also discussed how extreme conditions or changing environments might increase the chances for polyploids to survive. However, the local establishment and short-term persistence does not necessarily mean long-term evolutionary success. Despite the fact that most angiosperm lineages are likely to be paleopolyploids ${ }^{1-3}$ the number of ancient genome duplications in plants is still exceedingly rare. While some lineages have undergone one or two additional genome doublings, ${ }^{4,5}$ others do not seem to have undergone a single additional WGD in the past 100-150 million years ago (i.e. since the hexaploidy event is the common ancestor of most eudicots). Considering the abundance of present-day polyploids, one might argue that the number of ancient WGDs that we can detect in the genomes of angiosperms seems to be low. ${ }^{20}$ Our current understanding of ancient WGDs in angiosperms is still based on a limited number of fully sequenced genomes and EST collections, and further sequencing of plants from different clades will probably reveal more cases of ancient polyploidy. Nevertheless, it is also likely that most of the present-day polyploids will not survive in the long-term. One explanation might lie in the regular birth-and-death process of species. New plant species constantly arise and disappear, resulting in a gradual turnover of the vegetation on Earth, and the number of young extant species is likely to be much higher than the number of old extant species. ${ }^{37}$ An alternative explanation might be that, even after polyploids have become established and have attained a considerable population size and range, they are at a higher risk of extinction than that of diploids, and their mean longevity is shorter than that of diploids. As far as we know, there are very little data available to either support or refute this hypothesis. The origination rate, extinction rate, and longevity of species have been studied on several occasions. ${ }^{37-39}$ However, we do not know how these parameters differ between polyploids and diploids. One limitation is that for many lineages, we do not know the number and timing of polypoidy that occurred in the evolutionary past of that lineage. Wood et al. ${ }^{40}$ attempted to overcome this by estimating the number of ploidy-changes based on the distribution of chromosome numbers, although it is unclear how well their estimation matches reality as large chromosome number reductions can occur shortly after polyploidizations. ${ }^{41}$ Mayrose et al. ${ }^{42}$ devised a probabilistic model to infer the number of polyploidization events along a phylogeny based on chromosome numbers, and such efforts might help our understanding of the evolutionary process of polyploid-derived lineages in the absence of genomic data. Another complication is that the extinction rate is difficult to estimate, and tends to be rather hypothetical in many cases. ${ }^{38} \mathrm{~A}$ better understanding of the extinction rate of polyploids will undoubtedly enhance our understanding of their evolutionary dynamics.

It is also important to consider the different phases outlined in Figure 1 when discussing various characteristics of polyploids, and the differences between diploids and polyploids. The extinction rate and range distribution is likely to differ depending on the phase, and different factors might influence the success of polyploids in different phases. For instance, polyploids in their initial stages of establishment will often have a small population size and restricted distribution, and consequently a higher risk of extinction and smaller chance of long-term survival. ${ }^{21}$ This differs from new species that emerge as a result of gradual speciation caused by geographical isolation and potentially already have a large population size to start with. By contrast, polyploids in their latter phases are those that have managed to attain a considerable population size and range, and might have different characteristics and a lower risk of extinction, although we do not know how their extinction rates would compare with that of diploid species. Also, some characteristics that are advantageous for their short-term success might not be advantageous for their long-term evolutionary success. Indeed, although switching to asexual reproduction or selfing might favor the rapid establishment of a polyploid, such reproductive strategies would often result in reduced genetic variability, which might not be advantageous for surviving under fluctuating environments. On the other hand, having a larger genetic variation within the population might allow adaptation to a wider range of environments which could be crucial for expanding their range and surviving in the long-term, especially as low genetic variation can be a limiting factor for the distribution of a species..$^{43}$ Also, having an entire extra set of genes might give the polyploid lineage a long-lasting potential

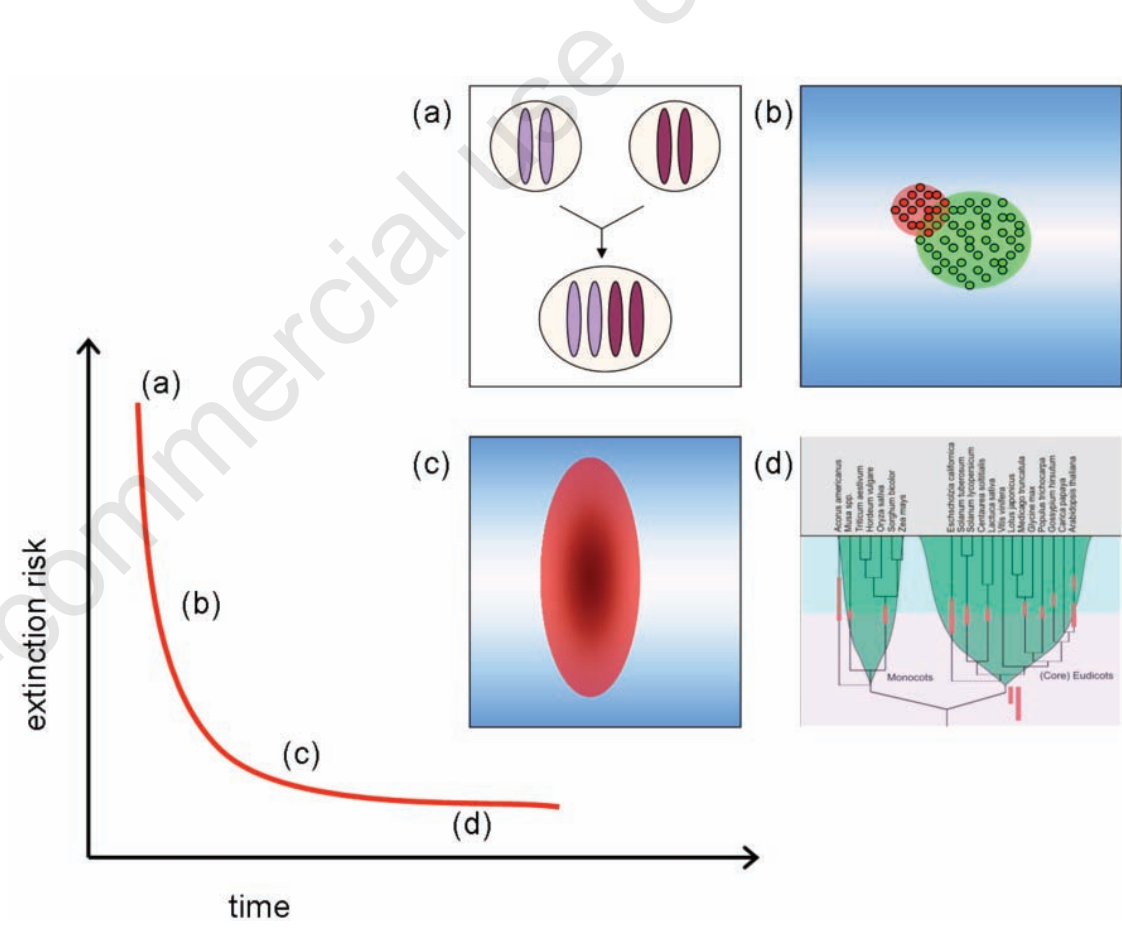

Figure 1. The road to evolutionary success. (a) The first step is the formation of individual polyploids, which is followed by (b) their establishment. A polyploid population is shown in red, and their diploid parental population is shown in green. (c) The next step is the persistence of polyploids. The distribution of a polyploid species is shown by the red oval, while the gradient in blue conceptually represents different environments. A newly established polyploid species often faces a high risk of extinction in its early stages, and must broaden its distribution and adapt to different environments in order to survive and avoid extinction. (d) Some polyploids go on to attain long-term evolutionary success and give rise to clades that survive for tens of millions of years, such as the polyploidization events that occurred in the evolutionary past of various angiosperm lineages (depicted by red bars). Many of the ancient polyploidizations seem to have occurred around the Cretaceous-Tertiary (KT) boundary (light-purple background represents the Cretaceous period, light-blue the Tertiary period), which is -65 million years ago (see ${ }^{50}$ for more details). Due to a background extinction rate, a given species can go extinct at any time due to various factors such as competition or climatic changes. The gradient of the extinction curve is hypothetical, and it remains to be tested whether the curve differs between diploid and polyploid lineages. See text for details. 
for adaptation and diversification. ${ }^{21,44,45}$ Thus, comparative studies of polyploids and diploids regarding factors such as their range, adaptability, or diversification potential is likely to be affected by the different phases of the sampled polyploids. Recent studies on the range or diversification rate have treated polyploids of all ages equally, ${ }^{34,46}$ and taking into account the different stages of establishment could be one of the next steps in order to imoprove our understanding of the evolutionary dynamics of polyploids.

Here we have considered the long-term evolutionary success of polyploids simply by their survival, regardless of the degree of their subsequent diversification. It is nevertheless worth discussing the potential link between polyploidy and species-richness or diversification $^{5,40,47,48}$ because species richness and diversity are often good indicators of the survivorship of a clade..$^{37}$ Although it has frequently been suggested that WGDs might be correlated with species-richness and can result in a radiation of species, some studies have failed to find an increase in species-richness or speciation associated with polyploidy. ${ }^{40,46}$ Meyers and Levin ${ }^{49}$ suggested that the abundance of polyploids or polyploid-derived lineages in plants can be explained by a simple mathematical model by which polyploids accumulate because it is an irreversible mutational process, and their hypothesis may serve as an ideal null model when evaluating the diversification of polyploid-derived lineages. However, many of these discussions might still be missing some crucial aspects. First, although recent studies have accounted for the phylogenetic history of diploids and polyploids, they have not considered their different distribution and ranges, or the different possible phases of polyploids (Figure 1). The range of a species is considered to be a major indicator of its ability to avoid extinction and diversify. If polyploids in their early phases when their ranges are still limited are included, as opposed to those in the latter phases when they are well-established and have propagated, this could be a confounding factor when comparing the survivorship and diversification rate of diploids and polyploids. Second, as Crow and Wagner ${ }^{50}$ argued, adaptation of polyploids and increased diversification is a process that occurs in response to, or is determined by, various ecological processes. Although polyploidization does not always result in a radiation of species, it is likely that ecological opportunities (e.g. available niches) will be a limiting factor. The potential for adaptation and diversification is likely to manifest itself when confronted with the need to adapt, such as in response to environmental changes, or when an ecological opportunity arises. Thus, rather than simply asking whether polyploids, in general, are associated with an increased species-richness compared to diploids, perhaps a more relevant question would be how do diploid and polyploid lineages differ in the way they adapt and radiate when presented with the same ecological pressure and opportunity.

\section{Success of ancient polyploids and periods of climatic change}

If indeed polyploids have a higher chance of becoming established under rough or changing environments, compared to normal, stable conditions, we would expect a longterm and global period of such conditions to result in a larger frequency of polyploids that do become locally established, which would in turn result in a larger frequency of established polyploids that do attain long-term evolutionary success. Another important aspect associated to periods of climatic change is the number of empty niches created due to many existing species becoming extinct by not being able to adapt to the changing environment. ${ }^{20}$ This would give the opportunity for newly established polyploids, or those that were already established but confined to limited localities, to propagate and expand their habitats and in some cases become dominant players in the new ecological system resulting in a successful radiation of species. ${ }^{45}$

Although it is apparent that polyploids succeeded in attaining long-term evolutionary success in various different lineages in the evolutionary history of angiosperms, ${ }^{4,5,48}$ determining the timing of the origin of ancient polyploids is by no means a trivial task, and the conditions and environments surrounding the formation and establishment of ancient polyploids are often largely unknown. Two recent studies proposed links between ancient WGDs and periods of climatic change. ${ }^{51,52}$ Fawcett et $a .^{51}$ dated many ancient WGDs in different plant families to approximately 60-70 million years ago, a time period overlapping the Cretaceous-Tertiary (K-T) boundary. This period is characterized by catastrophic events leading to global changes in the environment, resulting in an ecological upheaval and major disturbance of the vegetation. ${ }^{53,54}$ Mass extinction events, such as the K-T extinction event, are known to create new evolutionary opportunities, and post-extinction rebounds are thought to play a crucial role in changing the course of evolution..$^{55}$ The recovery of the vegetation after the K-T boundary probably took a million years or more in several localities ${ }^{56}$ providing many opportunities for various lineages to colonize new niches and proliferate in the new ecosystem. Also, the number of new species per se that originated after the extinction event could have increased, and it has been shown by studies on marine animals that the longevity of genera that originated shortly after the extinction event was longer on average than those that originated during periods not associated with extinction events. ${ }^{39}$

We argued earlier that limited ecological opportunities might restrict the radiation of a given lineage, and that this could be a confounding factor when comparing the diversification potential of polyploids and diploids because they would not have had the opportunity to realize their full diversification potential. However, these limitations are largely offset in periods of mass extinction due to climatic change. In such periods, the opportunity for both polyploids and diploids to adapt to fluctuating environments and colonize new niches would be ample, and they are more likely to be able to realize their full diversification potential. It is worth noting that some of the most species-rich families in plants (e.g. Poaceae, Solanaceae, Fabaceae), which are thought to have given rise to a large number of species after the K-T extinction event, seem to have undergone polyploidizations close to the time of the K-T extinction event (note that not all lineages underwent polyploidizations around this time period, e.g. the lineages of grapevine and papaya ${ }^{1,2}$ ). Although data to systematically compare the diversification rates of lineages that did experience a WGD around this period to those that did not are still limited, this might indeed underlie the large potential for polyploids to diversify, adapt, and occupy new niches.

Couvreur et al..$^{52}$ provided a comprehensive phylogeny and divergence dates of the Brassicaceae. The origin of this plant family was dated to approximately 37 million years ago, and an accelerated rate in diversification was detected from approximately 32 million years ago in the core Brassicaceae. It is thought that a drastic global cooling that occurred approximately 33 million years ag $0^{57}$ resulted in the extinction of several 'moister' clades and an increase in deciduous/dryadapted flora in Europe. ${ }^{58}$ The authors suggested that the Brassicaceae family originated as a tropical/subtropical family under a warm and humid climate, and that the evolution of some key characters and adaptation to the more arid and cool climate might have enabled them to radiate, whereas other tropics-adapted Brassicaceae probably went extinct due to the global cooling. ${ }^{52}$ The authors went on to propose that the WGD thought to have occurred approximately $30-40$ million years $\operatorname{ag}^{51,59}$ in the evolution of Brassicaceae might have contributed to their successful adaptation and radiation, and that the increase of new available niches laid the platform for the polyploid lineage to undergo rapid adaptive radiations and survive in the 
long term.

\section{Concluding remarks and future perspectives}

Here, we have discussed several factors attributed to the success of polyploids under harsh conditions and periods of climatic change, and highlighted aspects related to the long-term evolutionary success of polyploids. Although the significance of polyploidy in the evolution of angiosperms has been discussed for decades,,${ }^{6,811,12,36}$ we are only now beginning to understand its actual impact. A further increase in the number of genomic sequences and improvements in dating methods is likely to result in a better understanding of the timing of each ancient WGD in terms of its phylogenetic placement and ecological context. ${ }^{4}$ This should allow us to link the accumulating knowledge on recent polyploids and ancient, cryptic polyploids, and understand the impact of polyploidy in a broader evolutionary context. It must be noted though, that several different genetic, ecological, physiological, and morphological aspects have been associated with polyploidy, underlying the potential and also the complexity of polyploids. ${ }^{60}$ Identifying how these various possible factors interact with each other and contribute to the evolutionary success of polyploids will also become crucial.

\section{References}

1. Jaillon 0, Aury J, Noel B, et al. The grapevine genome sequence suggests ancestral hexaploidization in major angiosperm phyla. Nature 2007;449:463-7.

2. Tang H, Bowers JE, Wang X, et al. Synteny and collinearity in plant genomes. Science 2008;320:486-8.

3. Tang H, Bowers JE, Wang X, et al. Angiosperm genome comparisons reveal early polyploidy in the monocot lineage. Proc Natl Acad Sci USA 2010;107:472-7.

4. Van de Peer Y, Fawcett JA, Proost S, et al. The flowering world: a tale of duplications. Trends Plant Sci 2009;14:680-8.

5. Soltis DE, Albert VA, Leebens-Mack J, et al. Polyploidy and angiosperm diversification. Am JB 2009;96:336-48.

6. Thompson JD, Lumaret R. The evolutionary dynamics of polyploid plants: origins, establishment, and persistence. Trends Eco Evol 1992;7:302-7.

7. Otto SP. The evolutionary consequences of polyploidy. Cell 2007;131:452-62.

8. Comai L. The advantages and disadvantages of being polyploid. Nat Rev Genet 2005;6:836-46.

9. Ramsey J, Schemske DW. Pathways, mech- anisms, and rates of polyploid formation in flowering plants. Ann Rev Ecol Syst 1998; 29:467-501.

10. Husband BC. Constraints on polyploid evolution: a test of the minority cytotype exclusion principle. Proceedings of the Royal Society of London. Series B: Biological Sciences 2000;267:217-23.

11. Grant V. Plant speciation. New York, USA: Columbia University Press; 1981.

12. Stebbins GL. Polyploidy, hybridization, and the invasion of new habitats. Annals of Missouri Botanical Garden 1985;72:82432.

13. Hegarty M, Hiscock S. Polyploidy: doubling up for evolutionary success. Curr Biol 2007;17:R927-9.

14. Soltis PS, Soltis DE. The role of hybridization in plant speciation. Ann Rev Plant Biol 2009;60:561-88.

15. Brochmann C, Brystin AK, Alsos IG, et al. Polyploidy in arctic plants. Bio J Linn Soc 2004;82:521-36.

16. Parisod C, Holderegger R, Brochmann C. Evolutionary consequences of autopolyploidy. New Phytol 2010;186:5-17.

17. Bretagnolle F, Thompson JD. Gametes with the somatic chromosome number: mechanisms of their formation and role in the evolution of autopolyploid plants. New Phytol 1995;129:1-22.

18. Mable BK. Breaking down taxonomic barriers in polyploidy research. Trends Plant Sci 2003;8:582-90.

19. Barringer BC. Polyploidy and self-fertilization in flowering plants. Am J Bot 2007;94: 1527-33.

20. Van de Peer Y, Maere S, Meyer A. The evolutionary significance of ancient genome duplications. Nat Rev Genet 2009;10:72532.

21. Abbott RJ, Lowe AJ. Origins, establishment and evolution of new polyploid species: Senecio cambrensis and S.eboracensis in the British Isles. Bio J Linn Soc 2004;82: 467-74.

22. Dynesius M, Jansson R. Evolutionary consequences of changes in species' geographical distributions driven by Milankovitch climate oscillations. Proc Natl Acad Sci USA 2000;97:9115-20.

23. Gaeta RT, Pires JC, Iniguez-Luy F, et al. Genomic changes in resynthesized Brassica napus and their effect on gene expression and phenotype. Plant Cell 2007;19:3403-17.

24. Madlung A, Masuelli RW, Watson B, et al. Remodeling of DNA methylation and phenotypic and transcriptional changes in synthetic Arabidopsis allotetraploids. Plant Physiol 2002;129:733-46.

25. Adams KL, Cronn R, Percifield R, et al. Genes duplicated by polyploidy show unequal contributions to the transcrip- tome and organ-specific reciprocal silencing. Proc Natl Acad Sci USA 2003;100:464954.

26. Ha M, Kim E, Chen ZJ. Duplicate genes increase expression diversity in closely related species and allopolyploids. Proc Natl Acad Sci USA 2009;106:2295-300.

27. Riddle NC, Birchler JA. Effects of reunited diverged regulatory hierarchies in allopolyploids and species hybrids. Trends Genet 2003;19:597-600.

28. Oswald BP, Nuismer SL. Neopolyploidy and pathogen resistance. Proc Bio Sci2007;274:2393-7.

29. Soltis PS, Soltis DE. The role of genetic and genomic attributes in the success of polyploids. Proc Natl Acad Sci USA 2000; 97:7051-7.

30. Felber F. Establishment of a tetraploid cytotype in a diploid population: Effect of relative fitness of the cytotypes. J Evol Bio 1991;4:195-207.

31. Li B, Xu X, Ridout MS. Modelling the establishment and spread of autotetraploid plants in a spatially heterogeneous environment. J Evol Biol 2004;17:562-73.

32. Baack EJ. To succeed globally, disperse locally: effects of local pollen and seed dispersal on tetraploid establishment. Heredity 2005;94:538-46.

33. Rausch JH, Morgan MT. The effect of selffertilization, inbreeding depression, and population size on autopolyploid establishment. Evolution 2005;59:1867-75.

34. Martin SL, Husband BC. Influence of phylogeny and ploidy on species ranges of North American angiosperms. J Ecol 2009; 97:913-22.

35. Buggs RJA, Pannell JR. Ecological differentiation and diploid superiority across a moving ploidy contact zone. Evolution 2007;61:125-40.

36. Soltis DE, Soltis PS, Tate JA. Advances in the study of polyploidy since Plant speciation. New Phyt 2003;161:173-91.

37. Alroy J. Colloquium Paper: Dynamics of origination and extinction in the marine fossil record. Proc Nat Acad Sci USA 2008; 105(Suppl):11536-42.

38. Magallón S, Sanderson MJ. Absolute diversification rates in angiosperm clades. Evolution 2001;55:1762-80.

39. Miller AI, Foote M. Increased longevities of post-Paleozoic marine genera after mass extinctions. Science 2003;302:1030-2.

40. Wood TE, Takebayashi N, Barker MS, et al. The frequency of polyploid speciation in vascular plants. Proc Natl Acad Sci USA 2009;106:13875-9.

41. Lysak MA, Cheung K, Kitschke M, et al. Ancestral chromosomal blocks are triplicated in Brassiceae species with varying chromosome number and genome size. Plant Physiol 2007;145:402-10. 
42. Mayrose I, Barker MS, Otto SP. Probabilistic models of chromosome number evolution and the inference of polyploidy. Syst Biol 2010;59:132-44.

43. Kellermann V, van Heerwaarden B, Sgrò $\mathrm{CM}$, et al. Fundamental evolutionary limits in ecological traits Drive Drosophila species distributions. Science 2009;325: 1244-6.

44. Kejnovsky E, Leitch IJ, Leitch AR. Contrasting evolutionary dynamics between angiosperm and mammalian genomes. Trends Ecol Evol 2009;24:572-82.

45. Edger PP, Pires JC. Gene and genome duplications: the impact of dosage-sensitivity on the fate of nuclear genes. Chromosome Res 2009;17:699-717.

46. Vamosi JC, Dickinson TA. Polyploidy and diversification: a phylogenetic investigation in Rosaceae. Int J Plant Sci 2006; 167:349-58.

47. Otto SP, Whitton J. Polyploid incidence and evolution. Annu Rev Genet 2000;34:401-37.

48. De Bodt S, Maere S, Van de Peer Y. Genome duplication and the origin of angiosperms. Trends Ecol Evol 2005;20: 591-7.

49. Meyers LA, Levin DA. On the abundance of polyploids in flowering plants. Evolution 2006;60:1198-206.

50. Crow KD, Wagner GP. Proceedings of the SMBE Tri-National Young Investigators' Workshop 2005. What is the role of genome duplication in the evolution of complexity and diversity? Mol Biol Evol 2006;23:887-92.

51. Fawcett JA, Maere S, Van de Peer Y. Plants with double genomes might have had a better chance to survive the CretaceousTertiary extinction event. Proc Natl Acad Sci USA 2009;106:5737-42.

52. Couvreur TL, Franzke A, Al-Shehbaz IA, et al. Molecular phylogenetics, temporal diversification and principles of evolution in the mustard family (Brassicaceae). Mol Biol Evol 2010;27:55-71.

53. McElwain JC, Punyasena SW. Mass extinction events and the plant fossil record. Trends Ecol Evol 2007;22:548-57.

54. Schulte P, Alegret L, Arenillas I, et al. The
Chicxulub asteroid impact and mass extinction at the Cretaceous-Paleogene boundary. Science 2010;327:1214-8.

55. Erwin DH. Lessons from the past: biotic recoveries from mass extinctions. Proc Natl Acad Sci USA 2001;98:5399-403.

56. Johnson KR, Ellis B. A tropical rainforest in Colorado 1.4 million years after the Cretaceous-Tertiary boundary. Science 2002;296:2379-83.

57. Zachos J, Pagani M, Sloan L, et al. Trends, rhythms, and aberrations in global climate 65 Ma to present. Science 2001;292:68693.

58. Morley RJ. Interplate dispersal paths for megathermal angiosperms. Persp Plant Ecol Evol Syst 2003;6:5-20.

59. Schranz ME, Mitchell-Olds T. Independent ancient polyploidy events in the sister families Brassicaceae and Cleomaceae. Plant Cell 2006;18:1152-65.

60. Soltis DE, Burleigh JG. Surviving the K-T mass extinction: new perspectives of polyploidization in angiosperms. Proc Natl Acad Sci USA 2009;106:5455-6. 